\title{
Development of a Wind Tunnel DAQ System using LabVIEW Tools
}

\author{
A. A. Rodríguez-Sevillano ${ }^{1, a^{*}}$, M. A. Barcala-Montejano ${ }^{2, b}$, \\ S. Sacristán-Callejo ${ }^{3, c}$ \\ ${ }^{1,2}$ Departamento de Aerotecnia. Technical University of Madrid (Universidad Politécnica de \\ Madrid), Plaza del Cardenal Cisneros, 3. 28040 Madrid, Spain. \\ ${ }^{3}$ ExpalSystems. Madrid, Spain \\ aangel.rodriguez.sevillano@upm.es, ’miguel.barcala@upm.es, 'ssacristan@expal.biz
}

Keywords: DAQ based on LabVIEW, project based learning, bachelor degree project, wind tunnel design

\begin{abstract}
This paper presents the experimental three-year learning activity developed by a group of teachers in a wind tunnel facility. The authors, leading a team of students, carried out a project consisting of the design, assembly and testing of a wind tunnel. The project included all stages of the process from its initial specifications to its final quality flow assessments, going through the calculation of each element, and the building of the whole wind tunnel. The group of (final year) students was responsible for the whole wind tunnel project as a part of their bachelor degree project. The paper focuses on the development of wind tunnel data acquisition software. This automatic tool is essential to improve the automation of the data acquisition of the wind tunnel facility systems, in particular for a 6 DOF multi-axis force/torque sensor. This work can be considered as a typical example of real engineering practice: a set of specifications that has to be modified due to the constraints imposed throughout the project, in order to obtain the final result.
\end{abstract}

\section{Introduction}

The School of Aeronautics and Aerospace Engineering (Universidad Politécnica de Madrid) recognized the need to build a low turbulence wind tunnel for research and teaching [1-3]. The wind tunnel would be mainly dedicated to study, characterize and quantify the aerodynamic behavior of different models under controlled conditions for research and teaching purposes. A group of researchers and teaching staff realized that this engineering project could be used as a project based learning activity to be developed by the students as a part of their compulsory final year project. Up to now, the team of students and teaching staff has completed the design and construction of the tunnel [4], as well as its measurement equipment facilities. Currently, the wind tunnel is being improved and updated with new instrumentation. The first results obtained a wind speed at the test section close to $50 \mathrm{~m} / \mathrm{s}$. At the same time, the mean turbulence intensity level is below 0.5 . As well as describing the design and building process, the wind tunnel instrumentation [5] and the LabVIEW data acquisition tool will also be discussed. This paper also describes the objectives of using LabVIEW for this acquisition system, the structure of this software instrument and its weakest and strongest points (cost, compatibility, user-friendliness, etc.) for this application. This solution, developed by an undergraduate project, provides the basic structures and main lessons to teach students in a workbench programming environment [6-11] in instrumentation system.

\section{DAQ using LabVIEW}

Computers provide a highly efficient environment for developing new systems that help to carry out repeated tasks in laboratory tests. The new wind tunnel facility has been provided with a complete acquisition system related to the forces and torques on the test model, ambient conditions data and airspeed inside the tunnel test section. LabVIEW environment is a graphic programming language used to build and run this software application. Its quality as a virtual workbench makes LabVIEW a good choice to expose students to data measurement system based on a programming 
language without any other hardware needed except a computer. In addition, National Instruments supplies a wide range of I/O cards, easy plug-in setup thus allowing for the study of programming structures without the need of install hardware. Low-cost cards specific for development and student tasks are included in National Instruments products.

One 6 DOF Force \& Torque sensor, an environmental indicator sensor with ambient humidity, temperature and pressure data and a differential manometer for airspeed measure are included in the LabVIEW data acquisition system. This software also supplies a file with all data measurements and a quick summary of test data in the user-interface panel.

\section{Wind Tunnel}

With approximately a one square meter section in the test chamber (Fig. 1, on the left) and four fans installed in the exhaust area (Fig. 1, on the right), the wind tunnel facilities provide an airspeed up to $50 \mathrm{~m} / \mathrm{s}$ in the test section with a very low turbulence intensity levelof 0.1 . This aerodynamic facility allows teachers and researchers to increase their involvement in new projects, and to test new developments and ideas. It also provides an important area to collaborate with private businesses for aerodynamic studies and help keep technological knowledge developed at the university as relevant as possible to the business sector's needs. The shortage of laboratory space limited the dimensions of the design. Due to this, the wind tunnel decided upon was a suction and open-circuit type.

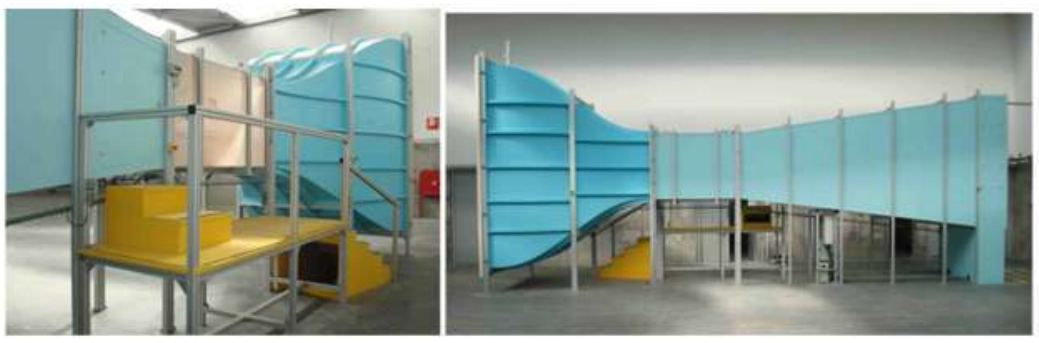

Figure 1: Wind tunnel facility

The structure is mainly built of wood and aluminum frames and the wind tunnel has a glass window for viewing the tests chamber. Inside this test area, on the floor, the attitude angle change assembly is installed. It consists of a two-movable-bars system to change the pitch and yaw angle of the models inside the test section. Once the angle has been set, several screws hold it in the desired attitude during the test phase. For the time being, this assembly is hand-operated (Fig. 2, on the left), but a new motor-operated set will be implemented (Fig. 2, on the right).
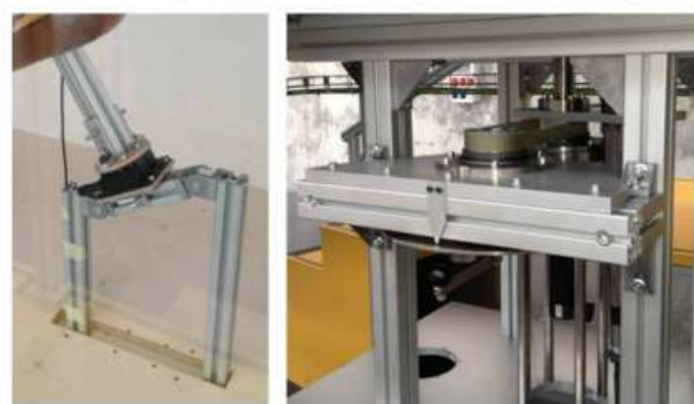

Figure 2: The two systems of changing model attitude

The four axial fans, which are responsible for the air flow inside the tunnel, are installed in the exhaust section. The wind tunnel uses a variable frequency control to set up the desired speed of the axial fans and to achieve the necessary speed in the test section. Although it is now hand-controlled, the fans' power box has an Input/Output (I/O) connection which means it can be software-controlled and integrated with a complete laboratory test software control system. Undoubtedly, this is one of the future stages for students in their projects. 
Turbulence Intensity Level. To determine the turbulence intensity level in the main flow direction, the instantaneous velocity signal, $\mathrm{u}_{\mathrm{i}}$ is expressed as:

$$
u_{i}=\bar{u}+u_{i}{ }^{\prime}
$$

which is the sum of a time-averaged component $\bar{u}$ and the turbulent fluctuation component, $u^{\prime}$. In short, the turbulence intensity level [12] is the ratio between the root-mean-square of the turbulent velocity fluctuations and the time-averaged velocity.

The linear displacement of the hot-wire probe has been was carried out with a linear guide. It is a TSR-1000B model from Zaber, the maximum displacement is $1,000 \mathrm{~mm}$, the position of the measuring probe being fixed with an accuracy of $\pm 68 \mu \mathrm{m}$. For velocity measurements, a Dantec Dynamics 54T42 MiniCTA hot-wire anemometer has been used. Velocity measurements start once the four fans'steady state has been reached. Then the hot wire probe moves along the $z$ axis in 10 mm steps, and at each measuring position the velocity is sampled at $1 \mathrm{kHz}$ for $10 \mathrm{~s}$. The hot-wire is placed in a vertical plane so that the velocity component in the plane normal to the wire direction is obtained. From the recorded samples, the mean velocity at each position, $U(x)$, as well as the turbulence intensity, $\mathrm{I}_{\mathrm{U}}(\mathrm{x})$ are calculated:

$$
I_{U}(x)=\frac{\sigma_{x}(x)}{U(x)}
$$

which $\sigma_{\mathrm{x}}$ is the standard deviation or root mean square value.

Measurement Sensors. The wind tunnel installation has five different probes with three individual transducers to send measurement signals to the data acquisition (DAQ) cards. The decision on which type of sensors and their number was taken in accordance with the requirements for testing and the teaching staff's criteria. At this point, this set of sensors is the Force and Torque (F\&T) measuring instrument, supplemented with test condition sensors. The system's components are the following:

- A six-degrees-of-freedom (6 DOF) force and torque silicon gage sensor (Fig. 3) for the force and torque measurements caused by flow around the model during tests. This sensor includes the transducer and signal amplification in the same system (six differential channels input)

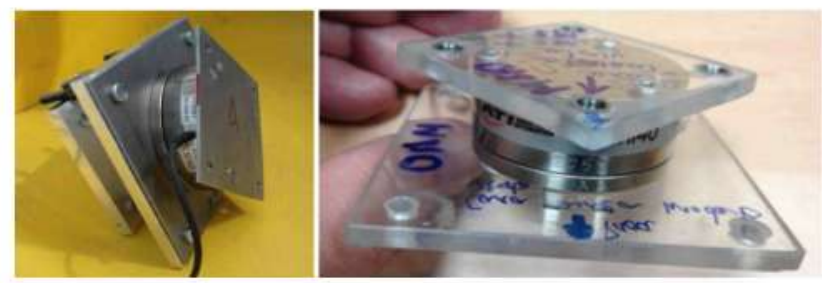

Figure 3: 6 DOF force and torque gage sensor (low and high measurement ranges)

- A pitot tube probe (Fig. 4, on the right) for airspeed in test section evaluation, which is wired to a differential pressure transducer (one differential channel input)

- Ambient temperature, pressure and humidity probes integrated in the transducer equipment (Fig. 4 , on the right). An environmental measurement unit (three differential channels input)

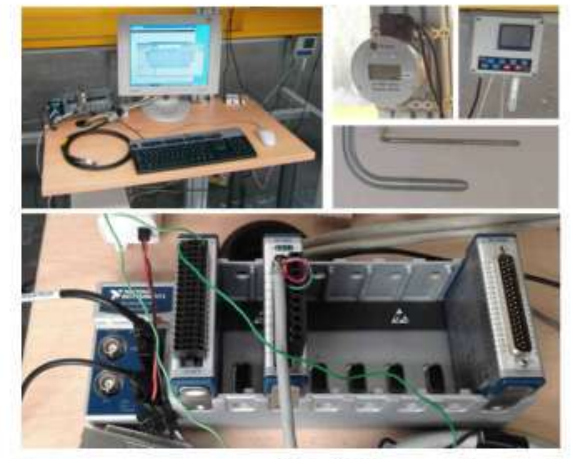

Figure 4: Acquisition and measurement (DAQ) cards and computer test equipment 
All the devices supply a voltage output signal, which is converted to a physical signal through their own constants inside LabVIEW software. Another important element of the systems is the data acquisition cards. All sensor signals need to be interpreted as digital signals, sent to the computer and saved for later data analysis. This is the task of the data acquisition cards (DAQ cards).

The first stage of this project consists of defining the needs of the acquisition system (channels, sample rate, samples, ranges, etc.) and choosing the hardware that meets the criteria. Once LabVIEW was chosen as the software environment, the hardware selected was a National Instruments (NI) data cards. National Instruments provides a wide range of data cards, modules and equipment for acquisition. Furthermore, its compatibility and full use of hardware capabilities are assured. Note that National Instruments is the parent company of LabVIEW. The best choice for the data acquisition was two independent DAQ cards, one for model test data and the other for tunnel and environment test conditions. Although this choice was more expensive than other alternatives, it provides more flexibility. One DAQ device records airspeed, ambient temperature, atmospheric pressure and relative humidity. These sensors are integrated within the tunnel facilities, regardless of the test type and model measurements. The other DAQ device records model data measurements. This device is a compact $\mathrm{DAQ}^{\circ}$ system (NI product) that consists of a chassis with eight slots for eight different DAQ cards (Fig. 4, on the bottom). Thus to change the F\&T sensor due to the expected forces on the model or because of the size of the prototype or to add a new card to control models with movable parts can be easily done, without modifying the tunnel measuring system. Both devices, which are connected via a USB cable, are set up for different sampling rates (samples/second), depending on the needs. The force and torque sensor, which experiences greater variation in its measurements due to fluctuation effects, usually require a higher sampling rate.

The students, under the supervision of teachers and researchers, fitted all connections, assemblies and wiring needed to connect the sensors to the corresponding transducers and acquisition cards used. They also carried out the selection of suitable measurement equipment and instrumentation for the wind tunnel facilities.

\section{The Software Choice}

The aim of this project was the selection and design of a complete software and hardware data acquisition system for a wind tunnel. This system must meet a set of initial requirements. The selection of the programming environment, and appropriate hardware, will not limit any possible improvementsto the wind tunnel in the future. The hardware choice has been detailed above and we will now discuss the software. The software acquisition environment was developed and updated by students as a part of their Bachelor degree project. This project focuses on the software programming to include new measurement modules and improve the application for easy test tasks. It also reduces the sensors-to-computer communication programming rules and user interface design (Fig. 5), without losing functionality. It should not be forgotten that the centerpiece of assessment for students is to increase the capabilities of the wind tunnel. Hence, to minimize problems, hardware-software integration difficulties must be assessed at all times to focus efforts on the objectives and divert attention away from additional complications.

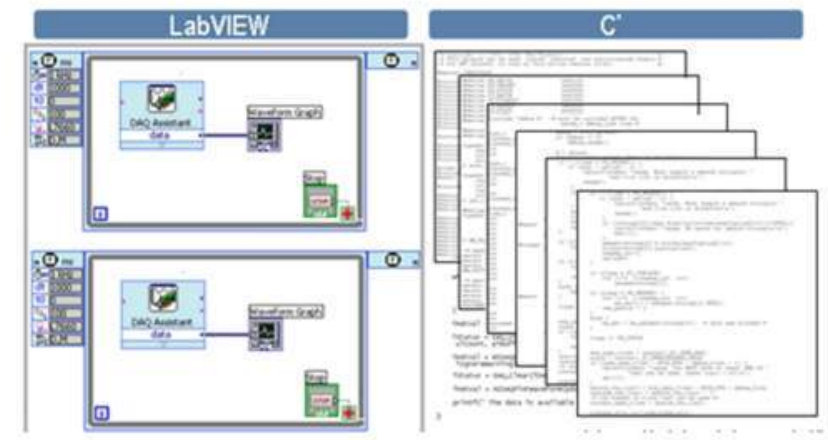

Figure 5: A comparison between visual programming language (LabVIEW) and a language based on sequential code text. (Source: www.ni.com) 
LabVIEW is one of the best DAQ software in terms of providing user-friendly functions and wizards. It is also a widespread programming language in academic, industry and research labs. Moreover, LabVIEW has a very wide network of forums and topics with a lot of examples and skilled developers to help solve queries. Another good characteristic of LabVIEW software is that a really complex user interface with a lot of different buttons, switches, state bars, controls and displays (Fig. 6) can be built into it without much effort, which makes the control panel userfriendly. It is also easy to create a graph display to plot and monitor a signal andit is not much more difficult to create alarm triggers either; and all this without losing acquisition (Input), control (I/O) and mathematical signal treatment capabilities. Besides, if NI hardware is selected, we can be completely confident that the DAQ cards will be detected by the computer and no signal communication problems will arise.

Notwithstanding the reasons above, one of the evaluation points when selecting the programming software lies with the students' skills and competencies. Most students involved in this project have no prior knowledge of a programming environment. Therefore, we needed to find user-friendly and easy to adapt software which does not require previous programming knowledge. Bearing in mind that students will only remain in the development process of the project while they carry out their bachelor degree project, it is necessary to use software that allows the students to quickly adapt to the given stage of the research project. For all these reasons and also the fact that the university had previously worked with LabVIEW environments, the project team leaders decided on National Instruments' LabVIEW programming environment.

Software Acquisition. The first task the students undertake in this section is the evaluation of the signals of the system, which are all signals that are going to be measured and recorded by the acquisition card. These signals are the following

- Six analog inputs for the $6 \mathrm{DOF}$ force and torque sensor.

- One analog input for the differential pressure transducer (airspeed).

- Three analog inputs for environmental unit: temperature, pressure and relative humidity

We now use the LabVIEW's wizard, which allows us to have full control over the functions used in software acquisition instrument. This wizard is very convenient for quickly evaluating systems. The program structure is divided into three big boxes: the acquisition module box, the recording module box, and the data processing module box. During the program development, we decided to include a pre-processing with LabVIEW's tools to record and show the raw signal, and also to record the processed signal. This includes the configuration of a low-pass filter and average value computing of some measurements. This processing module also includes the conversion of the volts signal into physical data and records it in another file. The programming environment is the user's interface panel (Fig. 6). This is the panel shown to the user, where the controls appear and where the graphic display power of LabVIEW is seen.

For this application we have organized the user interface panel into three different tabs. The first and principal one is the "Results monitor" (Fig. 6). This tab has two graphic displays to show the measured data, but it also has the controls to start the acquisition process, to reset the calibration system and to activate and deactivate data saving. In the second tab, we find all the data acquisition configurations such as sampling rate, number of samples, channels to acquire, etc. The last tab is for the data file saving options. Here, the name file can be changed and add any comment to the data file can be added. The reason to separate these functions in different tabs is to optimize the information offered to the user, to maximize the available space on screen and to prevent debug user errors. Taking advantage of the easiness LabVIEW offers in creating a display, and making the most out of the event and case structures, minimizes any errorswhich might occur while operating the software, by restricting information and control in different tabs. As well as these tabs, on the right of user's interface display is located the "Data Panel". This is a simple display where the user's averages of measured data and the translation of these volt measurements to a physical data are displayed 


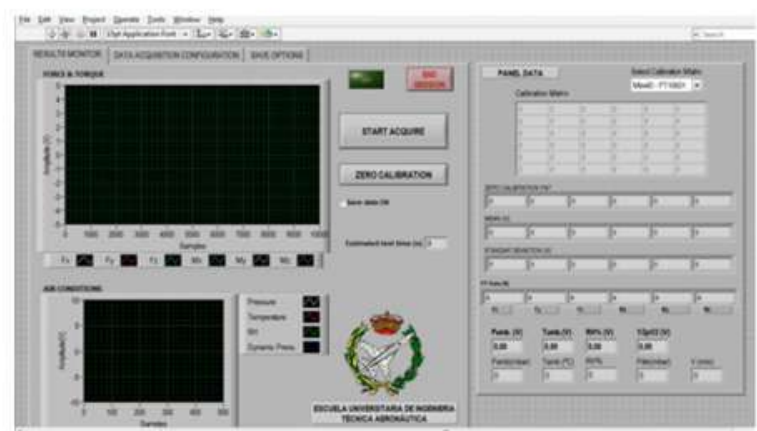

Figure 6: User's interface front panel, results monitor

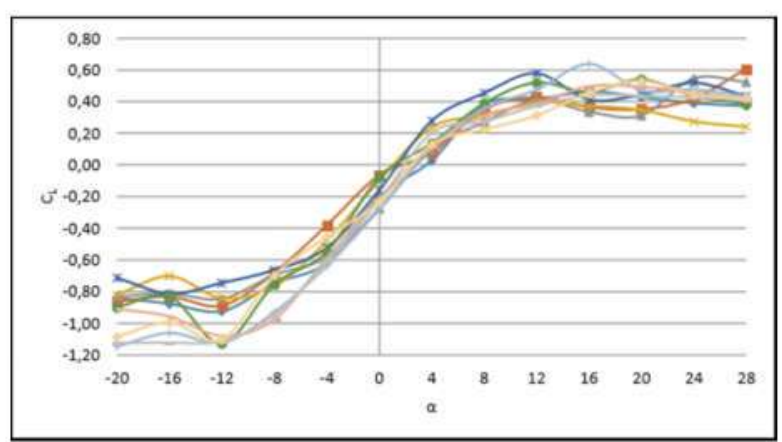

Figure 7: $\mathrm{C}_{\mathrm{L}}$ vs AOA. Three different tests for the same airfoil with the same test configuration

Software Assessment. We have managed to reduce to a third of the time required for each test from the first software version in comparison with previous equipment and methods. This process has a user-friendly interface and now all measurement data is centralized in a single computer. This is a more effective management of the stored data. Furthermore, we have developed a simple program to upload data files by generating multiple tests on the same configuration and automatically calculate the mean value of: aerodynamic coefficients, air velocity in the test section, forces experimented by the model, and even graphs. The processing time has been greatly reduced to approximately $10 \%$ of the original processing time; and what is more, we have removed up to $90 \%$ of the human errors in the process caused by doing repeated tasks.

System Validation. After the assembly of all components of the system and sensors, and also the completion of the software programming tasks, tests were performed to check the sensors operating individually. In addition to these tests, the calibrations were tested. After finishing the integration tests, the software is run with all sensors several times, to ensure that all cards and sensors work and that the software saves the data to files properly. The next step is to evaluate the results and validate them in two different ways. The first one is the comparison of the results obtained in the tunnel with international wind tunnel databases, and the second one is to evaluate the repeatability of the data measured. A wing airfoil was tested in the wind tunnel to check the values acquired with results obtained for the same airfoil in other aerodynamic facilities. The results are quite promising.

The combination of hardware and software used has achieved a high level of repeatability in the experimental results. The same airfoil as above was tested at different airspeeds, changing the angle of attack (AOA), and the tests repeated several times for each configuration. In this way we have assessed that the data obtained for the same test configuration, but at different times, are very similar (Fig. 7).

Industrial Application. The same LabVIEW software has been used with slight modifications within the industry as a data acquisition system. For this application, the software only includes an air velocity and tri-axial force and torque sensors. The project involves Research and Development (R\&D) Department of EXPAL Systems (whose parent company is MAXAM) in the study of a guidance kit on a high-speed blower. The data obtained were used to size and design aerodynamic fins, to adapt the kit to maximize its performance.

In this application, the LabVIEW software was linked to a very high range force $\&$ torque sensor (up to $1 \mathrm{kN}$ measuring range), with a sensitivity of $0.5 \mathrm{pC} / \mathrm{N}$ (about $0.01 \mathrm{~N}$ ). The forces generated by the fins of the guidance kit (Fig. 8) are not as big as to require a sensor with this range. But the whole test stand includes a strong arm to support the kit against the supersonic flow. This arm and the front section of the guidance kit (about $4 \mathrm{~cm}^{2}$ ), are responsible for generating higher drag forces in tests in high speed conditions. Also, the need to characterize the fins demanded a high sensitivity to the smallest force effects. 


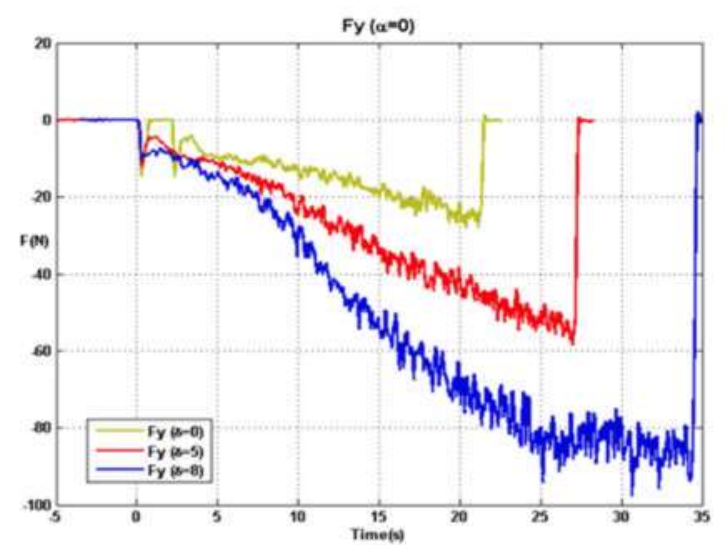

Figure 8: Test data of a guidance kit configuration. Force versus time, as airspeed increases, $\mathrm{AOA}=0$. (Source: EXPAL Systems)

\section{Students' Feedback}

The preparation of this system has involved the work of students in the final stage of their degree. This work provides a facility that will support many students in their Bachelor degree projects. The decision to use LabVIEW software has been very successful in the work of students. As well as encouraging the students to learn something that is not included in the curriculum, the improved testing method means that students and researchers who conduct their studies here make better use of their time spent on testing.

\section{Planning for the Future}

The software outlines discussed above are an evolving program, which changes over time to include enhancements and new tunnel systems. It is equally important to mark some lines of future choice for testing new systems. One of those is the assembly of a motorized system that enables attitude angle changes in the model and the addition of this control in software testing. On the other hand, another important and interesting improvement is the challenge to automatically control and from the computer the tunnel airspeed. This control also allows feedback directly to the air velocity data that the tunnel already has. It would be interesting to extend conventional static models to the study of models with movable parts such as rotor blades, folding wings and other systems, to change their configuration during the test trial.

\section{Conclusions}

The team of lecturers considers that the project has achieved the two main goals of the project. The first one is to involve the students in a research team, with highly complex tasks, and relevant engineering decisions. This project has been developed in one of the most relevant aeronautical tasks, that is, wind tunnel research. The students have shown a high level of motivation throughout the project. The second goal was to carry out their final Bachelor degree project. We have shown a part of these results in this paper.

\section{Acknowledgements}

The team of lecturers would like to thank to all the people who have contributed with their efforts and knowledge to the wind tunnel design and construction, and to the flow quality testing: P. Porcar, J.M. Moya, P. Razorla, S. Greppi, M. Tchimchilka, S. Navarro, R. Parreño, C. Villamil, F. Gandía, and JM. Holgado. 


\section{References}

[1] Duit R, Treagust DF. Conceptual change: A powerful framework for improving science teaching and learning. International journal of science education. 2003;25(6):671-88.

[2] Adams R, Evangelou D, English L, Figueiredo AD, Mousoulides N, Pawley AL, et al. Multiple perspectives on engaging future engineers. J Eng Educ. 2011;100(1):48-88.

[3] Niedderer H, Budde M, Givry D, Psillos D, Tiberghien A. Learning process studies. In: Contributions from science education research. Springer; 2007. p. 159-71.

[4] Abbaspour M, Shojaee M. Innovative approach to design a new national low speed wind tunnel. International Journal of Environmental Science \& Technology. 2009;6(1):23-34.

[5] Braun EM, Lu FK, Panicker PK, Mitchell RR, Wilson DR, Dutton JC. Supersonic blowdown wind tunnel control using LabVIEW TM. Proc. 46th AIAA aerospace sciences meeting and exhibit, american institute of aeronautics and astronautics; ; 1801.

[6] Ertugrul N. Towards virtual laboratories: A survey of LabVIEW-based teaching/learning tools and future trends. International Journal of Engineering Education. 2000;16(3):171-80.

[7] Hennessey R, Loya H, Diong B, Wicker R. LabVIEW-based automatic control systems laboratory using local and remote experimentation approaches. Proc. NI week conf. and expo., austin, TX, USA; ; 2000.

[8] Higa ML, Tawy DM, Lord SM. An introduction to LabVIEW exercise for an electronics class. Frontiers in education, 2002. FIE 2002. 32nd annual; IEEE; 2002.

[9] Imbrie P, Raghavan S. Work in progress-A remote e-laboratory for student investigation, manipulation and learning. Frontiers in education, 2005. FIE'05. proceedings 35th annual conference; IEEE; 2005.

[10] Pecen R, Salim M, Zora A. A labview based instrumentation system for a wind-solar hybrid power station. Journal of Industrial Technology $2004 ; 20(3): 1-8$.

[11] Rapuano S, Zoino F. A learning management system including laboratory experiments on measurement instrumentation. Instrumentation and Measurement, IEEE Transactions on. 2006;55(5): 1757-66.

[12] Rodríguez-Sevillano A, Pérez-Grande I, Meseguer $\mathbf{J}$. On the onset of turbulence in natural convection on inclined plates. Exp Therm Fluid Sci. 2011;35(1):68-72. 